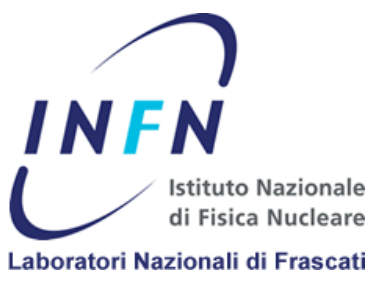

SPARC-BD-07/002

April 2007

\title{
GENERATION OF SHORT THZ BUNCH TRAINS IN A RF PHOTOINJECTOR
}

\author{
M. Boscolo ${ }^{\mathrm{a},{ }^{*}}$, M. Ferrario ${ }^{\mathrm{a}}$, , C. Vaccarezza $^{\mathrm{a}}, \mathrm{I}_{\text {. Boscolo }}^{\mathrm{b}}$, F. Castelli ${ }^{\mathrm{b}}$, S. Cialdi $^{\mathrm{b}}$ \\ ${ }^{a}$ INFN/LNF, Via Enrico Fermi 40, 00044 Frascati (Roma), Italy \\ ${ }^{\mathrm{b}}$ University and INFN/MI, Via Celoria 16, 20133 Milano, Italy
}

\begin{abstract}
A radiofrequency electron gun joined to a compressor can generate trains of $\mathrm{THz}$ subpicosecond electron pulses by illuminating the photocathode with trains of laser pulses. Assuming a prompt electron emission, each laser pulse generates an electron disk at the cathode. The disk train evolves toward a slightly modulated slug having a peculiar sawtooth energy modulation. This kind of energy modulation is transformed into a density modulation within a velocity bunching compressor recovering at a good extent the initial multi-disk profile. The electron beam evolution is analyzed through simulations looking at its features as function of pulse and frequency characteristics of the laser and of the accelerator parameters.
\end{abstract}

PACS: 29.25.Bx; 29.27.Bd

Keywords: electron pulse trains, laser, photoemission, rf-gun, Space-charge, rf compressor

In publication: in Nuclear Inst. and Methods in Physics Research A http://dx.doi.org/10.1016/j.nima.2007.04.129

${ }^{*}$ Corresponding author. Tel.: +39-0694032636; fax: +39-0694032256. E-mail address: manuela.boscolo@lnf.infn.it (M. Boscolo) 


\section{INTRODUCTION}

Electron pulse trains of some hundreds $\mathrm{pC}$ charge, sub-picosecond length and repetition frequency of some $\mathrm{THz}$ can be useful to drive FEL experiments, plasma accelerators and efficient generation of $\mathrm{THz}$ radiation [1,2]. In this paper we discuss the possibility to produce such a high frequency and high charge train in a radio-frequency electron gun by illuminating the photocathode with a comb-like laser pulse, thus generating subpicosecond high charge disk trains. In this configuration the electrons in each disk experience a large longitudinal space charge field with a linear correlation along the bunch. As the micro-bunches travel along the gun and the downstream drift they expand longitudinally $[3,4]$. The work done by the space charge force produces an energy modulated electron beam with a sawtooth profile of the energy modulation with $\Delta \mathrm{E} \sim 0.4 \mathrm{MeV}$. This energy modulated electron beam can be transformed into a density modulated electron beam in a magnetic or rf compressor device.

The amplitude of the energy modulation depends on the number and initial thickness of the electron micro-bunches. Given the charge per macrobunch, the thinner the microbunches the higher the resulting energy spread at the end of the rf-gun and, in turn, the tighter turns the current modulation after a compression. We present simulations of comb beams having both sinusoidal and Gaussian waveforms and different number of pulses $(2 \leq \mathrm{N} \leq 10)$. The intent is to determine the beam characteristics in term of spatial shape, energy and density modulation.

The beam dynamics have been modeled by simulations with PARMELA [5] code. We present and discuss separately the electron bunch evolution inside the rf-gun and downstream inside a velocity-buncher. We outline a possible scheme of the optical apparatus tailored to the generation of laser pulse trains.

\section{BEHAVIOUR OF AN ELECTRON BEAM IN AN RF-GUN DRIVEN BY A LASER PULSE TRAIN}

\subsection{The electron gun}

As a reference injector we have consider the SPARC machine [6,7] even though it does not necessarily represent the best layout for the laser-comb operational mode; some room for additional optimization is left for a future work.

The SPARC parameters relevant for our study are listed in Table 1. The rf-gun of Fig. 1 has a 1.6 cell operating at S-band with a peak field on the cathode of $120 \mathrm{MV} / \mathrm{m}$ followed by an emittance compensating solenoid [8]. The crystal copper photocathode is excited by light pulses at $266 \mathrm{~nm}$ third harmonic of Ti:Sa laser delivering $0.5 \mathrm{~mJ} /$ pulse. We consider the electric and magnetic operational parameters chosen for the nominal working point of the SPARC injector, that is the working condition of the space-charge emittance compensation regime [8] matched for the $1 \mathrm{nC}$, $10 \mathrm{ps}$ long flat top electron bunch. The behaviour is studied as function of the number of pulses within the train (i.e. the train frequency), the charge and the peak current in each pulse. The charge per micropulse $\mathrm{Q}_{\mathrm{P}}$, given the pulse number $\mathrm{N}$ and the charge of the macropulse $\mathrm{Q}_{\text {tot }}$, is $\mathrm{Q}_{\mathrm{P}}=\mathrm{Q}_{\text {tot }} / \mathrm{N}$. The peak current is determined by the micropulse duration. 

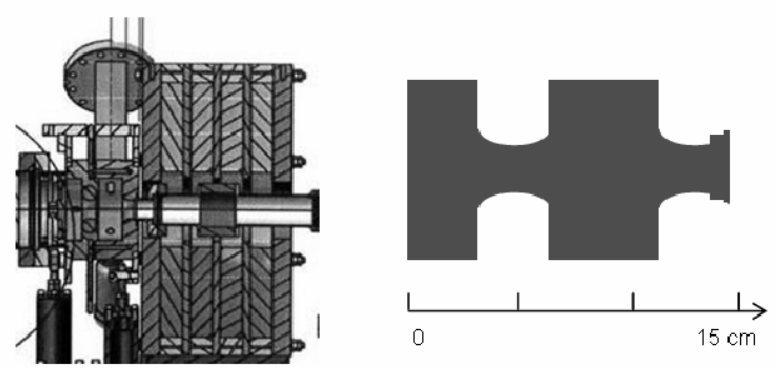

Fig. 1. SPARC RF gun layout: the left drawing is the mechanical design with the magnetic coil, the right drawing shows the 1 and $1 / 2$ rf-cavities of the gun.

Table 1. Nominal parameters for the rf-gun (first column), velocity bunching cavity (second column) and two accelerating cavities (third and fourth column) in the SPARC accelerator.

\begin{tabular}{|c|c|c|c|c|}
\hline & rf-gun & I TW section & II TW section & III TW section \\
\hline Gradient $[\mathrm{MV} / \mathrm{m}[$ & 120 & 25 & 12.5 & 12.5 \\
\hline Energy [MeV] & 5.6 & 17 & 55 & 88 \\
\hline Phase $\phi[\mathrm{deg}]$ & 32 & $-99 /-101$ & 0 (on crest) & 0 (on crest) \\
\hline $\begin{array}{l}\text { Solenoid field } \\
\text { [Gauss] }\end{array}$ & 2730 & 615 & 0 & 0 \\
\hline length $[\mathrm{cm}]$ & 15 & 300 & 300 & 300 \\
\hline
\end{tabular}

\subsection{Evolution of sinusoidal shaped electron beam within the rf-gun}

We report in this section the outputs (see Fig. 2) of the simulation obtained for $\mathrm{N}=4$ pulses only, as the results and considerations relative to this case can be extended to any other number $\mathrm{N}$ providing a non-negligible density modulation.

In Fig. 2 the evolutions of (a) the current modulation, (b) the energy modulation and (c) the spatial shape of the electron bunch from the cathode up to the end of the rf-gun are presented, respectively, in the first, second and third rows.

Current modulation washes out almost completely at the end of the drift at $150 \mathrm{~cm}$; the energy modulation of each micropulse increases up to the end and, remarkably, assumes the peculiar sawtooth fashion; the electron beam transverse dimension increases notably (within the rf section) and then decreases roughly up to the original size with a small wiggle. The maximum of energy modulation sets in the center between the pulses.

Simulations for different values of N (and with constant total charge and initial sinusoidal laser modulation) show that the higher $\mathrm{N}$ the lower is the energy modulation, as shown in Fig. 3 . We see that at $\mathrm{N}=10$ the energy modulation is less than $100 \mathrm{KeV}$, which still allows a non-negligible density modulation within a compressor (see below). 


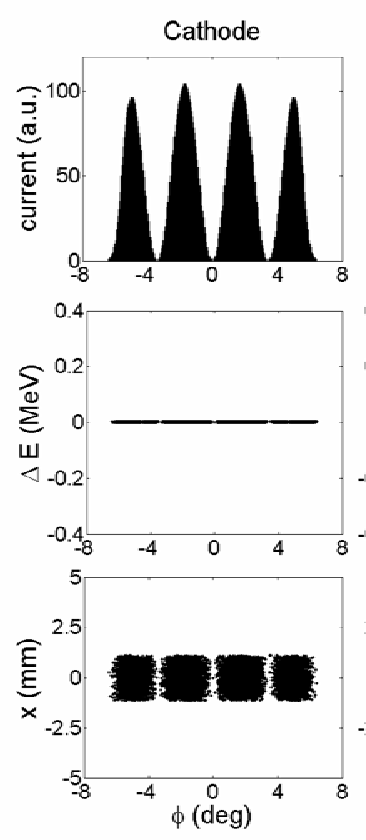

(a)
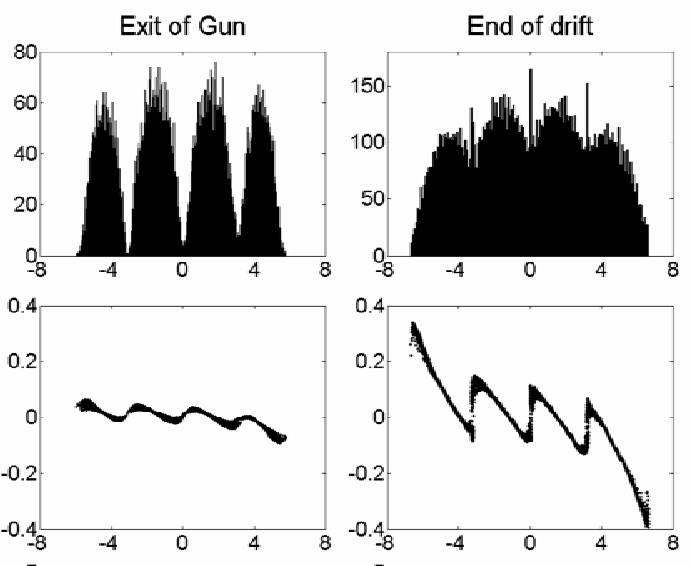

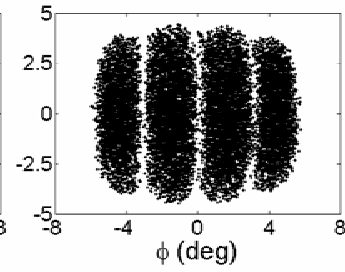

(b)

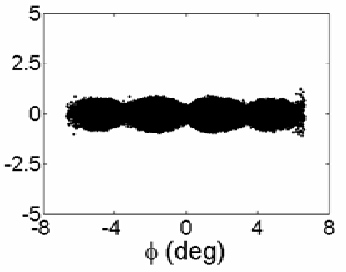

(c)

Fig. 2: Evolution of a train of four bunches spanning $10 \mathrm{ps}$. The rows from top refer respectively to longitudinal profile, energy modulation $\mathrm{E}(\mathrm{MeV})$ and electron beam spatial configuration $\mathrm{x}(\mathrm{mm})$; columns from left refer respectively to (a) the cathode, (b) the exit of bare-gun and (c) the end of the drift at $150 \mathrm{~cm}$.

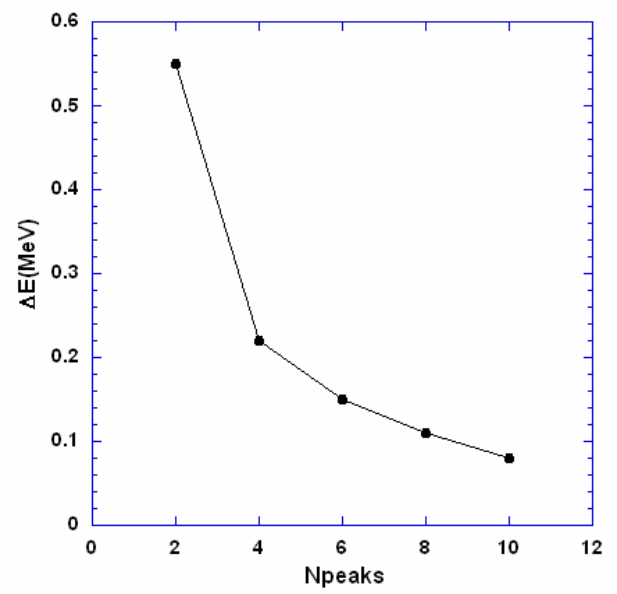

Fig. 3: Energy modulation $\Delta \mathrm{E}(\mathrm{MeV})$ at the exit of the rf-gun as a function of the number $\mathrm{N}$ of sinusoidal pulses.

\subsection{Evolution of Gaussian shaped comb electron beam within the rf-gun}

We present in detail the two $\mathrm{N}=2$ and $\mathrm{N}=6$ cases, the first one for its relevance and the second one as an example.

In the $\mathrm{N}=2$ case a simple Gaussian current modulation as that shown in Fig. 4 leads to an energy modulation $\Delta \mathrm{E}=0.6 \mathrm{MeV}$ which is enough for obtaining a relevant current modulation after compression. From the figure we see that two initial transverse cylindrical shaped pulses evolve towards two simil-ellipsoids with comparable transverse dimension. The slightly deformed ellipsoidal shape might be due to the mutual interaction between the two micro-bunches $[3,4]$. 
It is worth remarking that the spatial distance between the two pulses could be set at any required value. This possibility can be of some interest in pulse probe and in PWFA experiments [9].

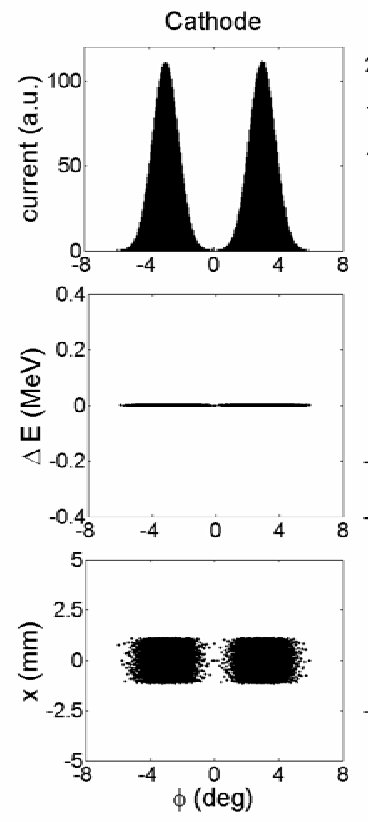

(a)

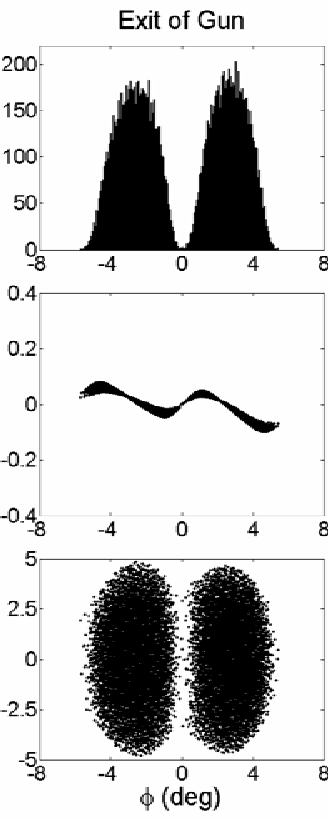

(b)
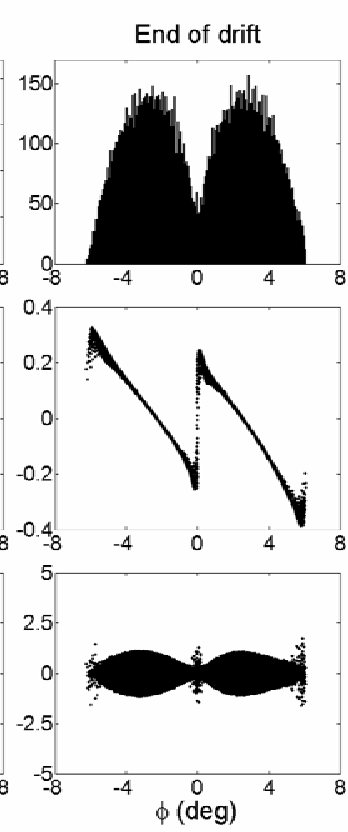

(c)

Fig. 4: Evolution of two bunches covering 10 ps interval. The rows from top refer respectively to longitudinal profile, to energy modulation $\Delta \mathrm{E}(\mathrm{MeV})$ and to electron beam spatial configuration $\mathrm{x}(\mathrm{mm})$; the column from left refer respectively to (a) the cathode, (b) the exit of gun and (c) the end of the drift at $150 \mathrm{~cm}$.

Simulations with $\mathrm{N}=6$ Gaussian pulses (see Fig. 5) show that the narrower the micro-pulse the higher the energy modulation and also the neater the energy separation between pulses.

(a)

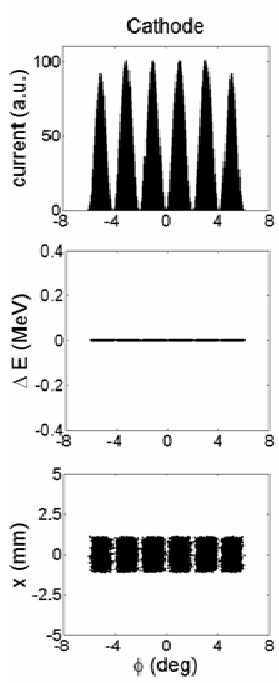

(b)

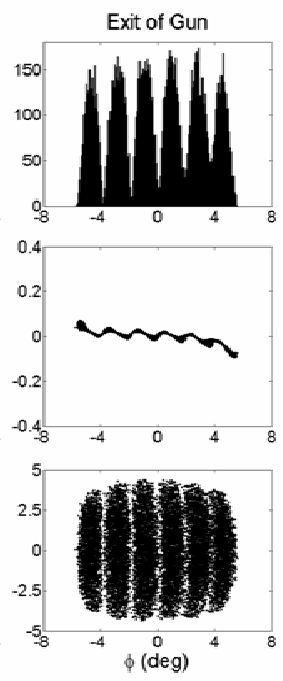

(c)
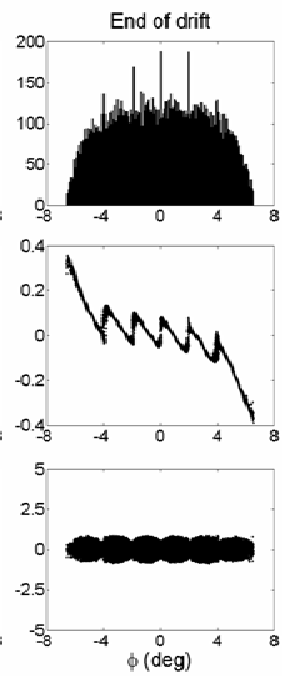

(a)
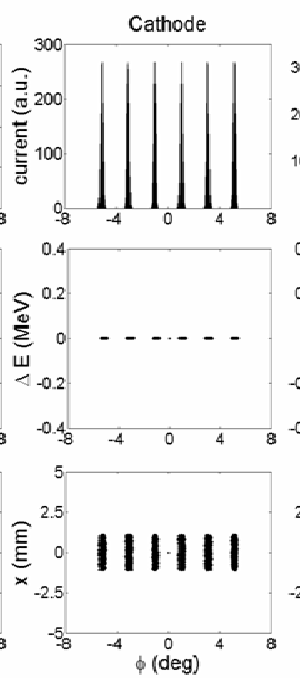

(b)
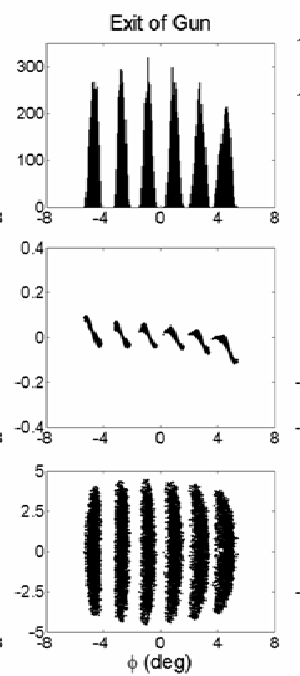

(c)
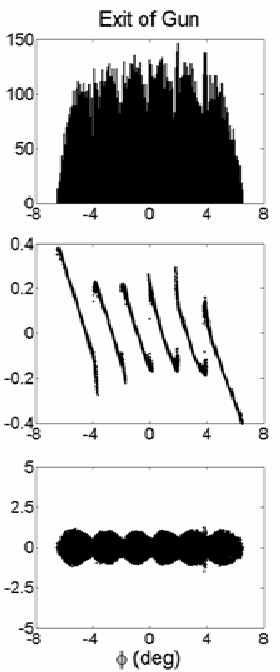

Fig. 5: Evolution of six bunches electron beam train: left frame refers to micro-bunches of FWHM $=1 \mathrm{ps}$, right frame of FWHM $=0.2 \mathrm{ps}$. The rows from top refer respectively to longitudinal profile, 
to energy modulation $\Delta \mathrm{E}(\mathrm{MeV})$ and electron beam spatial configuration $\mathrm{x}(\mathrm{mm})$; the columns from left refer respectively to (a) the cathode, (b) the exit of gun and (c) the end of the drift at $150 \mathrm{~cm}$.

\subsection{Evolution of the electron beam within the velocity-buncher and accelerator}

The electron beam after the rf-gun and drift enters a compressor, for transforming the energy modulation into current modulation, and then it enters an acceleration section. Its evolution is shown in Figs. 6, 7 and 8. A compressor in the SPARC machine is straightforwardly realized by properly setting the rf-phase and the value of the accelerating gradient of the first accelerating cavity (see the table 1) [10]. This type of buncher is called velocity-buncher. The task of the buncher is to rotate the array of the left bent segments shown in the longitudinal phase space (central raw of the right column in Figs. 2, 4 and 5) into an array of thin vertical segments. This result can be obtained in a velocity bunching compressor because of the rotation induced by its $\mathrm{rf}$ field in the longitudinal phase space, providing that the electron beam is set at the right rf phase.

The optimized injection phase must be lower than $-99^{\circ}$, in order to obtain the proper rotation in the phase space for all the $\mathrm{N}$ microbunches, as shown in the current and energy plots obtained from simulations for the $\mathrm{N}=6$ case and different input phases (see Fig. 7).
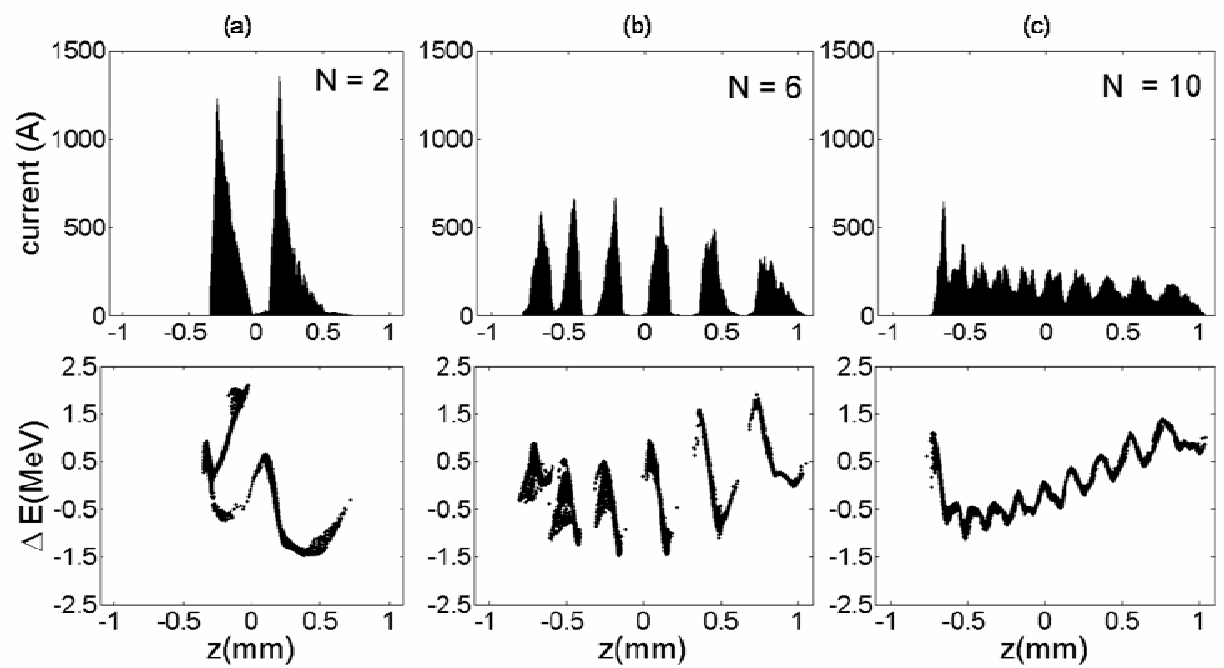

Fig. 6. The intensity modulated electron comb beam at the end of the accelerator for the three cases: (a) $\mathrm{N}=2$, (b) $\mathrm{N}=6$ and (c) $\mathrm{N}=10$. 
(a)
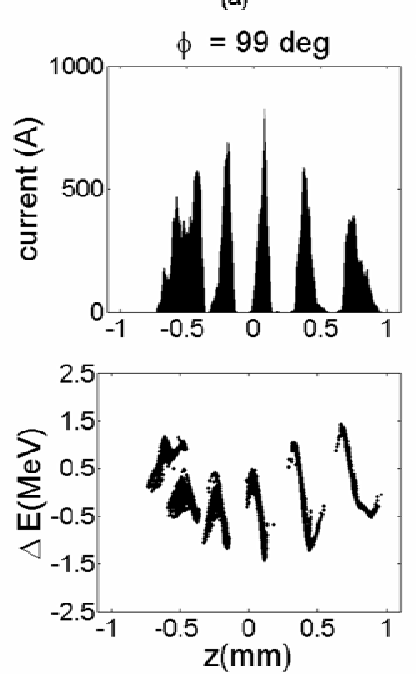

(b)
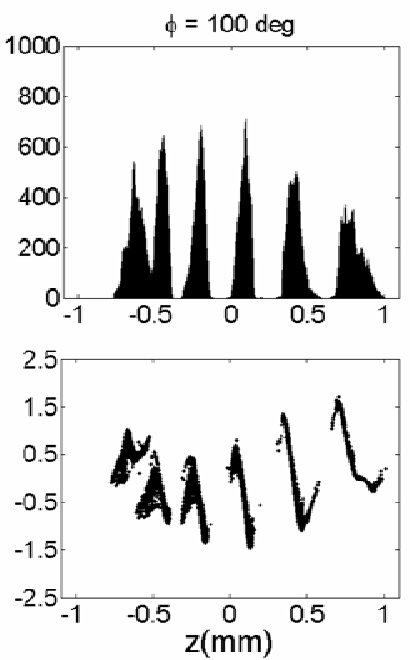

(c)
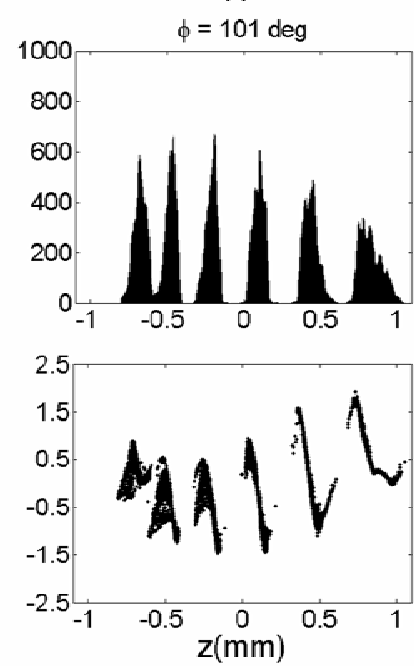

Fig. 7: Rf compression for $\mathrm{N}=6$ with rf-phase for the velocity buncher cavity of (a) $-99^{\circ}$ (b) $-100^{\circ}$ (c) $-101^{\circ}$. We see that the two leading micropulses move from a partial overlap to a complete separation decreasing the phase of only $2^{\circ}$.

We notice that each segment makes a rotation of a $(3 / 4) \pi$ angle about its center in phase space within the velocity buncher cavity, while the entire train makes a rotation of about $(5 / 4) \pi$. Because of these two rotations, the head-tail electrons interchange both in each micro-bunch and in the whole train. The large rotation angle of the entire train imposes the $-101^{\circ}$ injection phase so that all the electrons of the train are acted upon by a decelerating field. At this optimized injection phase $101^{\circ}$ the structure of the six micro-bunches comes out to be well defined with a high peak current $\left(\mathrm{Q}_{\mathrm{tot}}=1.1 \mathrm{nC}\right)$. Only the last pulse has different characteristics (risulting larger and lower).

The energy, emittance and current obtained for the two cases of 0.55 and $1.1 \mathrm{nC} \mathrm{nC}$ total charge, are shown in Fig. 8. The rms emittance of the pulses is calculated to be $7 \mu \mathrm{m}$ and $14 \mu \mathrm{m}$, respectively, the energy spread decreases from the first to the last pulse according to their relative initial phase and the currents are $\mathrm{I}_{\text {peak }} \sim 350 \mathrm{~A}$ and $\sim 700 \mathrm{~A}$ respectively (the pulses have different peak current).
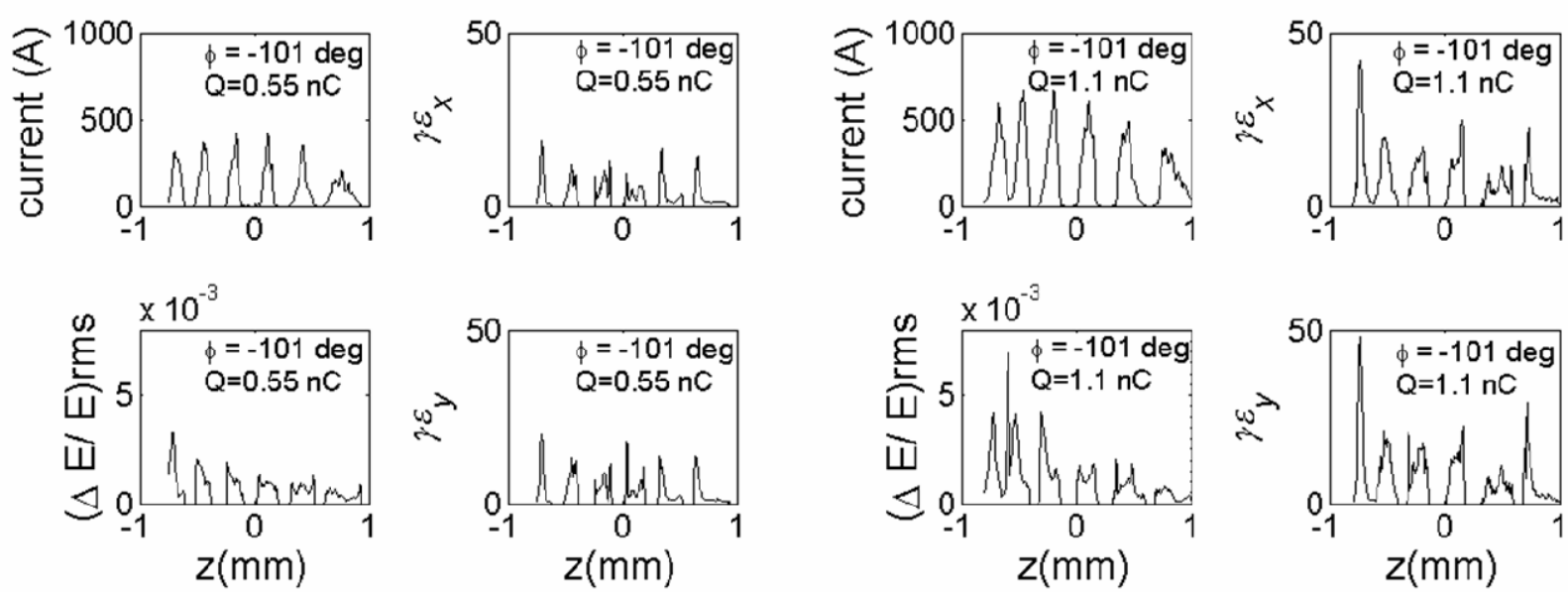

Fig. 8: The current and the emittance follow substantially the ratio between the total charge. The diagrams relative to $0.55 \mathrm{nC}$ (the four frames on the left side) do not have the large emittance 
deviation of the first pulse as shown in the diagrams relative to the $1.1 \mathrm{nC}$ (four frames on the right side).

The micro-bunches have a $\sigma_{z}$ of about $30 \mu \mathrm{m}$ with an inter-distance around $300 \mu \mathrm{m}$. The emittance substantially halves in passing the injection phase from $-96^{\circ}$ to $-101^{\circ}$ (except for the leading pulse). The electron beam evolves more cleanly for the case of $\mathrm{Q}=0.55 \mathrm{nC}$ than in the $\mathrm{Q}=1.1 \mathrm{nC}$ case. The investigation of the emittance variation versus the magnetic field of the compensating solenoids at the bunching and accelerating cavities has shown that its lowering is obtained at the expense of the density modulation. For example, it can be calculated that with $\mathrm{B}_{\text {gun }}=$ 2720 Gauss, $B_{1 \mathrm{TW}}=1500$ Gauss and $\mathrm{B}_{2 \mathrm{TW}}=1600$ Gauss the rms projected emittance is halved, but the current modulation is lost. At this stage, it seems that the reference set of parameters of the SPARC solenoids are a good trade off for the emittance and current modulation.

\section{LASER LAYOUT FOR PULSE TRAINS GENERATION}

Comb laser beams with different characteristics in terms of number and shape of the pulses can be produced with different optical layouts. The first observation is that the laser pulse used for driving the photocathode of the rf-gun is generated by a heavy amplification and, in cascade, by two/three harmonic up-conversions (depending if Ti:Sa or Nd:YAG(YLF) laser). The amplification does not allow a spiky input beam, hence the comb laser beam must be created in the UV region.

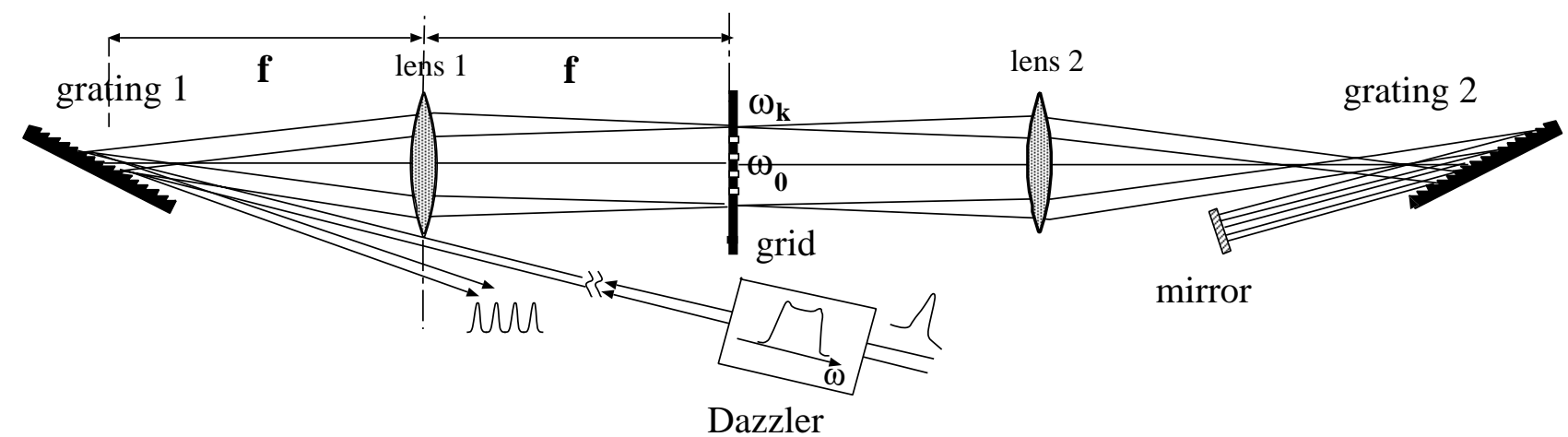

Fig. 9. Scheme of the $4 \mathrm{f}$ system arranged as a stretcher and with a spatial mask.

An optical system for generating a train up to four pulses is shown in Fig. 9. The system has a DAZZLER [11,12] which performs the shaping of the input pulse into a simil-rectangular pulse in the frequency domain and, in succession, a $4 \mathrm{f}$-system in the asymmetric configuration (that is arranged as a stretcher) [13] with a spatial mask at the Fourier plane. This apparatus with a mask made of a screen with four slits, as depicted in Fig. 9, generates four pulses as shown in the simulations of Fig. 10. A shaping optical system with an iris at the Fourier plane, instead of the DAZZLER, such that the wings of the laser pulse are cut down in the frequency domain [13] is able to generate the almost rectangular laser pulse as required for the generation of an array of pulses by means of a screen with slits. This arrangement without the DAZZLER is cheaper and easier but at the cost of losses and worse pulses. This very simple technique cannot be exploited for a higher number of pulses. 

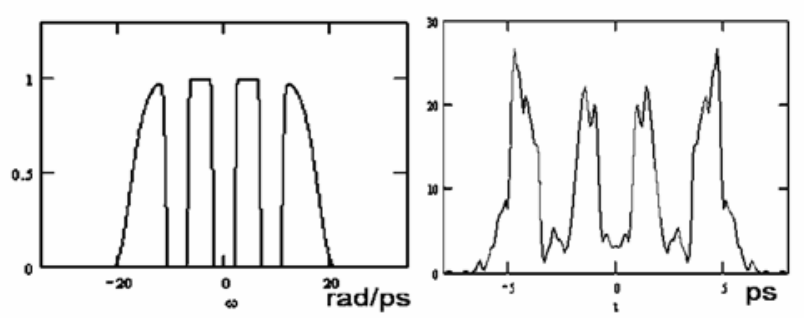

Fig. 10. Left frame: the pulse in the frequency domain, right frame: the simulated 4 output laser pulses.

The generation of an almost sinusoidal train can be easily done by means of the interference between two pulses in a Michelson interferometer, as shown in the scheme of Fig. 11.

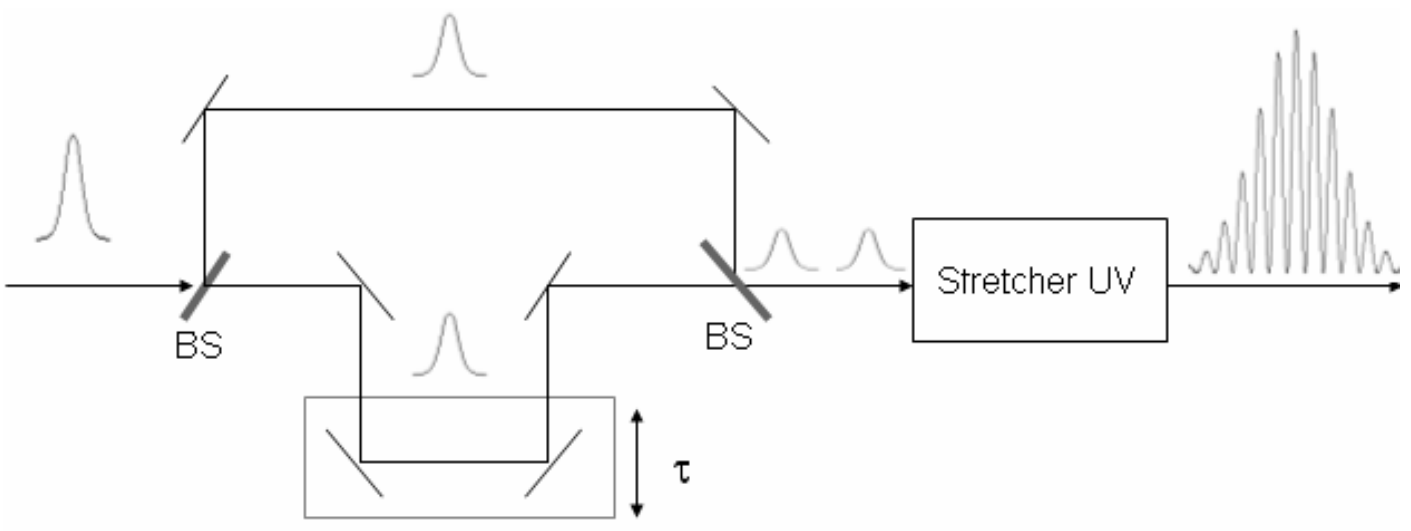

Fig. 11. Splitting of the input UV pulse into two pulses into a Michelson interferometer and recombination within the stretcher.

The mathematics is

$$
\begin{aligned}
& A_{\text {out }}(\omega)=A_{1}(\omega)+A_{2}(\omega)=\frac{1}{2} A_{\text {in }}(\omega)+\frac{1}{2} A_{\text {in }}(\omega) \cdot e^{i \tau \omega}=\frac{1}{2} A_{\text {in }}(\omega) \cdot\left(1+e^{i \tau \omega}\right) \\
& I_{\text {out }}(\omega)=\left|A_{\text {out }}(\omega)\right|^{2}=I_{\text {in }}(\omega) \cdot\left(\sin \left(\frac{\tau \cdot \omega}{2}\right)\right)^{2}
\end{aligned}
$$

The generation of a train with very narrow Gaussian pulses and of a targeted number of pulses can be done exploiting the spectral filtering technique. A pulse shape is the Fourier transform of the pattern transferred by the spatial filter onto the spectrum [14]. In our approach we use a spectral phase filtering: the phase response of the mask (filter) varies periodically with frequency, see Fig. 12. In this way the spectral bandwidth is divided in $\mathrm{N}$ sub-bandwidths whose distance in frequency is $\Delta \mathrm{F}$. Each radiation sub-bandwidth is conveyed into the relative pixel sub-array and will apply the same phase modulation. The repetition rate of the train is the frequency periodicity $\Delta \mathrm{F}$. The output intensity profile $\mathrm{I}(\mathrm{t})$ is related to the input waveform $\mathrm{E}(\omega)$ as

$$
I(t)=\left(\frac{1}{2 \pi}\right)^{2} \int e^{i \omega t} d \omega \int E^{*}\left(\omega^{\prime}\right) E\left(\omega^{\prime}-\omega\right) d \omega^{\prime}
$$




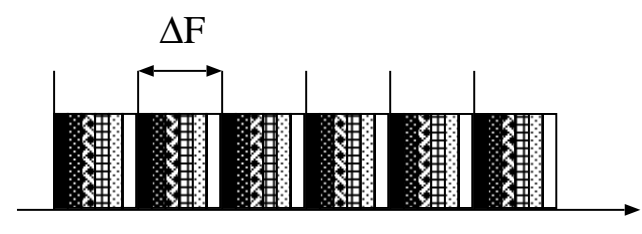

$\omega$

Fig. 12. Generation of a pulse train by a phase spectral filtering. The various shaded rectangles denote the pixels with different phases.

The intensity is the Fourier transform of the autocorrelation of the filtered spectrum. To obtain a set of pulses under a smooth envelope by using a phase filter, we must make a phase response with an autocorrelation that consists of a train of spectral pulses. Various pseudorandom phase sequences can provide this sequence.

We have tested with a pixellated mask the described technique. The pixels were grouped in bunches of 5 . The phase mask consisted of the repetition of the sequence [10010] where the logic 1 corresponded to $\Delta \varphi=0.7 \pi[15]$.

\section{DISCUSSION AND CONCLUSIONS}

We have analyzed the feasibility of a sub-picosecond electron bunch train in a rf-gun combined with a velocity buncher, exploiting both the capability of generating trains of subpicosecond laser pulses and the action of the space-charge in that system (which generates an energy modulation with a sawtooth profile). Simulations show the evolution from the initial multi pulse train into a spatial quasi-homogeneous beam developing the sawtooth profile energy modulation and the subsequent recovery of the spatial density modulation within an rf-accelerating structure operating in the velocity bunching mode.

The current profile of the micro-bunches turns out to be quite similar, except for the two extreme micro-bunches. This allows us to conclude that the intra-bunch action is almost negligible.

The final features of the electron bunch train are very sensitive to the injection phase into the rfcavity, to the charge of the macro-pulse and to the compensating solenoidal fields. The injection phase has to be around $-100^{\circ}$ because a phase space rotation of $270^{\circ}$ is required in order to obtain the whole compression. In this large rotation the rf-curvature shows its action on the macro-bunch tails, leading to both an enlargement and a shift of the two external current pulses. We remark that we have to work in an over-compressed regime in order to recover the original comb beam structure. The emittance value at the end of the accelerator depends almost proportionally on the charge and it is very sensitive to the rf phase of the buncher cavity. The first trials with different magnetic fields values have shown that there is a trade off between the amplitude modulation and the emittance. A more detailed analysis of the slice emittance compensation, that is the relevant parameter in this configuration, is in progress.

The feasibility of a comb electron beam is based on the possibility of producing trains of very narrow and powerful laser pulses. The spectral filtering and interferometric techniques are used to this purpose. About the laser pulse squeezing, it was tested that the damage threshold of a metal photocathode decreases fast with the decrease of the pulse width and it reaches few hundreds $\mathrm{GW} / \mathrm{cm}^{2}[16]$. By the way, the quantum efficiency increases with the increase of the intensity owing to a non-linear effect [16].

The investigation is performed with PARMELA code. With the electron beam parameters and electro-mechanical characteristics of that injector, we have found that the maximum number of 
density oscillations with an interesting depth is ten. The simulation on the six micro-bunches case with 200 fs initial width gives a peak current (with an amplitude) of about $750 \mathrm{~A}$ and a width of about $30 \mu \mathrm{m}$.

The simulations guided us into the dynamics of the modulated electron beam within the rf-gun. A multibunch electron beam with sub-ps bunches of very high current and targeted profiles opens interesting possibilities in plasma wake fields acceleration experiments and free electron lasers. In particular, a $\mathrm{THz}$ electron beam with high contrast opens the possibility of very efficient free electron laser in the far infrared region of the electromagnetic spectrum.

As a possible example for a quotation of emission in the $\mathrm{THz}$ range, we ran a simulation for a beam of $15 \mathrm{MeV}$ energy and got a current modulation higher than $50 \%$ with six microbunches with the laser modulation discussed above. The modulation wavelength results to be around $300 \mu \mathrm{m}$. Assuming, just for an evaluation, an undulator of parameter $\mathrm{K}=1$, which, consequently, would give a $27 \mathrm{~cm}$ long period and a number of periods $\mathrm{N}=10$, the coherent spontaneous power in the first harmonic calculated following ref. [17] is

$$
P_{\text {coh }} \approx \frac{\pi}{8} \frac{K^{2}}{1+K^{2}} \eta_{0} \frac{N^{2}}{\gamma^{2}} I^{2} A_{1}^{2} \cong 50 K W
$$

Here $\eta_{0}$ is the free space impedance, I the electron beam current and $A_{1}$ the amplitude of the first harmonic bunching. Incidentally, this coherent power is a factor two less than that evaluated with the formula reported in the Handbook of Accelerator Physics and Engineering [18].

We must remark that a radiating electron-beam-undulator system with a bunched electron beam behaves as a superradiant system because each microbunch but the last one is acted upon by the radiation emitted by the rear microbunches. This fact, that is the relatively high radiation emission due to the stimulation by the spontaneous emitted radiation, forces to put a properly tapered undulator in order to maintain the synchronism among the electron microbunches. The energy reduction per meter eEK $/ \gamma$ of the leading electron bunch, quoted from the motion equation, with $\mathrm{E}$ the electric field generated by the spontaneous coherent emission, results in a fraction of $\mathrm{MeV}$.

It is also worth noticing that a bunching as tight as it results from simulation allows efficient operation at higher harmonics. This operation is technically feasible because the parameters of the undulator relative to $\mathrm{THz}$ range are relaxed.

The calculations and considerations are a bit overestimated because the velocity buncher introduces a detuning among the electron bunches. A detailed behavior requires dedicated simulations. As a last observation, aiming to operate at a lower electron beam energy a magnetic buncher could be exploited in substitution of the above discussed velocity buncher, even if the beam characteristics could result worse than those provided by the velocity buncher.

\section{ACKNOWLEDGMENTS}

The work is partly supported by Ministero Istruzione Università Ricerca, Progetti Strategici, DD 1834, Dec.4, 2002 and European Contract RII3-CT-PHI506395CARE.

\section{REFERENCES}

[1] P.O.Shea et al., in: Proceedings of 2001 Particle Accelerator Conference, Chicago, IEEE, NJ, 2001, p.704.

[2] V. Stagno, G. Brautti, T. Clauser and I. Boscolo, Coherent radiation from Electrons in transverse Periodic Fields, Nuovo Cimento B 56 (1980) p. 219; I. Boscolo and V. Stagno, The Converter and the Transverse Optical Klystron, in: Nuovo Cimento B 58 (1980) p. 267.

[3] O.J.Luiten et al., Phys. Rev. Lett., vol 93 (2004) n. 9. 
[4] J.B. Rosenzweig et al., Emittance compensation with dynamically optimized photoelectron beam, Nucl. Instr. and Meth. A 557 (2006) 87.

[5] J.Billen, PARMELA, LA-UR-96-1835, 1996.

[6] D.Alesini et al., Status of the SPARC project, in: Proceedings of $10^{\text {th }}$ European Particle Accelerator Conference, Edinburgh, UK, 2006, p. 110.

[7] M.Boscolo et al., Laser comb: Simulations of pre-modulated e- beams at the photocathode of a high brightness rf photoinjector, in: Proceedings of $10^{\text {th }}$ European Particle Accelerator Conference, Edinburgh, UK, 2006, p. 98.

[8] L. Serafini, J.B. Rosenzweig, Phys. Rev. E 55 (1997) 7565.

[9] M. Boscolo, M. Ferrario, C. Vaccarezza, I. Boscolo, S. Cialdi, F. Castelli, , Int. J. Mod. Phys. B in publication in vol. 21 (2007).

[10] L. Serafini, M. Ferrario, Velocity Bunching in PhotoInjectors, AIP Conf. Proc. 581 (2001) 87.

[11] P. Turnois, Acoustic-optic Programmable dispersive filter for adaptive compensation of group delay time dispersion in laser systems, Opt. Comm. 140 (1997) 245.

[12]F. Verluise, V. Laude, J-P. Huignard, P. Turnois, A. Migus, Arbitrary dispersion control of ultrashort optical pulses with acoustic waves, J. Opt. Soc. Am. B 17 (2000) 138.

[13] S. Cialdi, M. Petrarca, C. Vicario and P. Musumeci, New scheme to produce high energy flat top laser pulses in UV, sent to Opt. Lett. for publication.

[14] A. M. Weiner, Femtosecond pulse shaping using spatial light modulators, Rev. Sci. Instrum. 71 (2000) 1929.

[15] I. Boscolo, S. Cialdi, F. Castelli, D. Cipriani, Report1-PHIN-CARE-JRA2-WP3, Second Task Pulse Shaping, http://www.infn.it/phin/docs files/19 Deliverable INFN Mi.pdf and references therein

[16] J.P.Girardeau-Montaut, C.Girardeau-Montaut, S.D.Moustaizis and C.Fotakis, "High current density produced by femtosecond nonlinear single-photon photoelectric emission from gold" Appl. Phys. Lett. 62 (1993) 426.

[17] I. Boscolo and V. Stagno, A Study of a Transverse Optical Klystron Experiment in ADONE (TOKA), Nucl. Instr. and Meth. 198 (1982) 483-496.

[18] Handbook of Accelerator Physics and Engineering, edited by A.W. Chao and M. Tigner, World Scientific. 